\title{
Predicting adult emotional and behavioral problems from externalizing problem trajectories in a 24-year longitudinal study
}

\author{
Joni Reef · Sofia Diamantopoulou $\cdot$ Inge van Meurs $\cdot$ \\ Frank Verhulst · Jan van der Ende
}

Received: 26 May 2009/ Accepted: 6 January 2010/Published online: 6 February 2010

(C) Springer-Verlag 2010

\begin{abstract}
The aim of this study was to examine the prediction of adult behavioral and emotional problems from developmental trajectories of externalizing behavior in a 24-years longitudinal population-based study of 2,076 children. The adult psychiatric outcome of these trajectories has not yet been examined. Trajectories of the four externalizing behavior types: aggression, opposition, property violations and status violations were determined separately through latent class growth analysis using data of five waves, covering ages $4-18$ years. We used regression analyses to determine the associations between children's trajectories and adults' psychiatric problems based on the Adult Self-Report. The developmental trajectories of the four types of externalizing behavior mostly predicted intrusive, aggressive and rule-breaking behavior in adulthood. Non-destructive behaviors in childhood such as opposition and status violations predict adult problems to a larger extent than destructive behaviors such as aggression and property violations. In general, children who develop through high-level trajectories are likely to suffer from
\end{abstract}

\footnotetext{
J. Reef $(\bowtie)$ - S. Diamantopoulou - I. van Meurs - F. Verhulst .

J. van der Ende

Department of Child and Adolescent Psychiatry,

Erasmus Medical Center-Sophia Children's Hospital,

P.O.Box 2060, 3000 CB Rotterdam, The Netherlands

e-mail: J.Reef@erasmusmc.nl

S. Diamantopoulou

e-mail: s.diamantopoulou@erasmusmc.nl

I. van Meurs

e-mail: i.vanmeurs@erasmusmc.nl

F. Verhulst

e-mail: f.verhulst@erasmusmc.nl

J. van der Ende

e-mail: j.vanderende@erasmusmc.nl
}

both internalizing and externalizing problem behavior in adulthood, regardless the direction of change (i.e. increasing/decreasing/persisting) of the high-level trajectory. We can conclude that the level rather than the developmental change of externalizing behavior problems has a larger impact on adult outcome.

Keywords Externalizing behavior . Child behavior checklist . Trajectory . Longitudinal development · Adult Self-Report

\section{Introduction}

To study externalizing behavior is of great importance because externalizing behavior causes many disturbances for the child and its social environment. Externalizing behavior in childhood and adolescence is highly predictive for later externalizing behavior [17, 18, 27] and other psychopathology [16, 37]. Because externalizing behavior can change both in form and severity over time [6, 9, 18, 29], studies on the development of externalizing behavior need to employ a developmental perspective including several measurement points to provide complete information about the severity of the problem. In addition, externalizing behavior problems comprise different types of behaviors that not only develop in different ways but also lead to different outcomes [6,15], consequently, developmental studies need to distinguish between development of different types of externalizing behavior [3]. In this 24-year follow-up study, we investigated the predictive strength of developmental trajectories in various childhood externalizing behavior types for a large range of adult psychopathology.

Because externalizing behavior comprises a large range of problem behaviors, Frick et al. [15] derived a 
classification through a meta-analysis of 44 studies. Four types of problem behaviors within the broader category of externalizing behavior were identified: property violations (e.g. lies, cruel to animals), aggression (e.g. fights, bullies), status violations (e.g. substance use, runaway) and oppositional (e.g. temper, stubborn). Only a few studies have confirmed this subdivision of externalizing behavior and have suggested that these different externalizing behavior types differ in their development and long-term outcome $[6,8,30,31]$. To our knowledge, no studies have reported about psychiatric outcomes in adulthood. Overall, status violations have been found to increase with increasing age [6] whereas the other subtypes decrease or persist over time. In addition, oppositional has been found to predict social problems in adulthood, while status violations has been found to predict both social problems and drug abuse, and aggression and property violations to predict drug abuse and risky sexual behavior $[8,30]$.

It is well established in the literature that the continuity of childhood externalizing behavior can follow different developmental trajectories over time. Developmental trajectories describe the course of behavior over time, that is, changes in both the level and the growth or decline of behaviors. It is important to know which change in level and growth across age may be considered normative for children and adolescents. Because, from both theoretical and clinical perspective, for defining abnormal behavior at any age point, it is indispensable to understand normal development. Previous longitudinal studies on the issue reported 'increasing', 'decreasing', 'stable high' and 'stable low' trajectories of externalizing behavior $[6,9,10,22]$. In addition to the level and the growth or decline of behaviors, individuals can start to display problem behavior at different ages [31]. As a result, some studies report a 'child onset' type, an 'adolescent onset' type [4, 19, 26], or a 'life-course persistent' and 'adolescence-limited' developmental trajectories of externalizing behavior. In the 'adolescent-limited' subgroup, externalizing behavior emerges in adolescence and desists after adolescence [9]. Considering these different developmental trajectories of externalizing behavior that groups of children follow, an average developmental trajectory that describes expected development for most children may be considered inadequate because of substantial variation around the mean. Therefore, it is important to consider groups of children that follow developmental trajectories that vary in level and shape. In the current study, we determined distinctive groups of individuals who are more likely to follow one developmental trajectory than another, within each type of externalizing behavior.

Different developmental trajectories have been found to have different adult outcomes [31]. For instance, children in stable high-level trajectories are more likely to show poor outcomes (e.g. delinquency, aggression or substance use) $[9,10,30]$ than children on low-level or increasing trajectories $[8,9,26]$. Furthermore, individuals in externalizing behavior trajectories of the 'life-course-persistent' and 'child onset' subtype experience in general more adult problems (e.g. delinquency and economic problems) than individuals in 'adolescent onset' and 'adolescent limited' trajectories [25, 26]. However, some studies fail to identify 'adolescent-limited' trajectories of externalizing behavior, or, report poor outcomes in adulthood for individuals who started to display problem behavior in adolescence [9, 25]. Evidently, both the type and the developmental course of externalizing behavior appear to widely differ among individuals and different types and developmental courses of externalizing behavior may lead to very different adult outcomes. Therefore, to be able to predict the adult outcome of externalizing behavior, different trajectories of distinct externalizing behavior types need to be examined.

In the present study, we investigated associations between developmental trajectories of externalizing behavior in childhood and a large range of psychiatric problems in adulthood. In a 24-year longitudinal population-based study, we examined the predictive value of trajectories of the four externalizing behavior types [15] (i.e., oppositional, aggression, property violations and status violations), based on five waves covering 4-18 years.

\section{Methods}

Sample

In 1983, a sample of 2,600 children from 13 birth cohorts aged 4-16 years was randomly selected from the general population of the province of Zuid-Holland in the Netherlands. Of each gender and age, 100 children were drawn from the municipal registers listing all residents in the province. A total of 2,447 parents could be reached, of whom $84.8 \%$ (2076 parents) completed the Child Behavior Checklist (CBCL) on their child. Parents were interviewed and completed the CBCL at 2-year time intervals in 1983, 1985, 1987, 1989 and 1991 [7]. Participants themselves were interviewed again in 2006 and 2007, when they were 28-40 years old.

Between January 2006 and June 2007 [32], we approached all participants from the original sample, except 23 who had died, 10 who were intellectually disabled, and 48 who had requested to be removed from the sample at an earlier stage of the study (see Fig. 1). We reached 1,791 of the 1,995 participants, 426 refused and 1,365 respondents provided usable data. The response rate in the data collection was $67 \%$ (1,365 of 2,043).

To investigate selective attrition, we performed logistic regression analyses to look at associations between age, 
Fig. 1 Flowchart of the data collection between 1983 and 2007

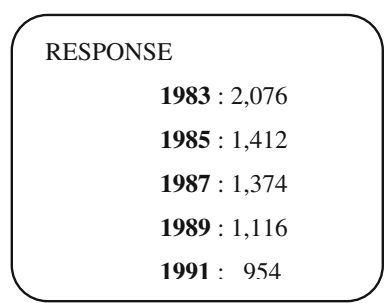

gender, socio-economic status (SES) and Total Problems Score of participants in 1983, and participation in 2006 and 2007. SES was scored on a six-step scale of parental occupation [33] with $1=$ lowest SES. Total Problems Score was calculated by summing 118 of the specific item scores on emotional and behavioral problems in the CBCL. Cut-off points for a deviant versus nondeviant Total Problems Score of the CBCL were based on the 85th percentile of the frequency distributions in the sample. Age, gender, Total Problems Score and SES were significantly associated with participation. Participation was more likely when participants were women (69.7 vs. $61.6 \%$; $\mathrm{OR}=1.44$; CI 1.20 $1.73 ; P<0.000$ ), if they were younger (mean age at baseline was 10.2 years for dropouts and 9.8 years for participants; $\mathrm{OR}=0.97$; CI $0.94-0.99 ; P<0.005)$, had a higher SES (3.4 for dropouts and 3.7 for participants; OR $=1.12$; CI 1.06-1.18; $P<0.000)$ and a nondeviant Total Problems Score at baseline $(17.3 \%$ for dropouts and $13.4 \%$ for participants; OR $=0.77$; CI 0.59-0.99; $P<0.043$ ).

\section{Measures}

\section{Externalizing behavior trajectories}

Externalizing behavior trajectories were based on assessment with the Child Behavior Checklist (CBCL) [1]. The $\mathrm{CBCL}$ is a questionnaire intended for completion by parents of 4- to 18-year-old children; it contains 120 items covering behavioral or emotional problems that have occurred during the past 6 months. The items are scored on a three-point scale: 0 (not true), 1 (somewhat or sometimes true) and 2 (very true or often true). The reliability and validity of the CBCL [1] have been confirmed for the Dutch version [35].

Externalizing behavior items of the CBCL which content showed a good match to the original items described by Frick [6, 15], were subdivided in four types: aggression, opposition, property violations, and status violations. The 21 items were used to create a score on these four types (see Table 1). A good fit of the four clusters was confirmed with a confirmatory factor analyses (the average goodness-of-fit index (GFI) was 0.92 for males and 0.96 for females; [6]).

Trajectories of externalizing behavior for ages 418 years were identified in a previous study on the ZuidHolland data (see Fig. 2). A semi-parametric, group-based
Table 1 Item description of the four externalizing behavior types

\begin{tabular}{ll}
\hline Frick cluster & Child behavior checklist item \\
\hline Aggression & Cruelty, bullying, or meanness to others \\
& Gets in many fights \\
& Physically attacks people \\
& Threatens people \\
Opposition & Argues a lot \\
& Disobedient at home \\
& Disobedient at school \\
& Stubborn, sullen, or irritable \\
& Sulks a lot \\
& Teases a lot \\
& Temper tantrums or hot temper \\
Cruel to animals & Lying or cheating \\
Property violations & Sets fires \\
& Steals at home \\
& Steals outside the home \\
Vandalism & Runs away from home \\
Status violations & Truancy, skips school \\
& Uses alcohol or drugs for not medical purposes \\
& \\
&
\end{tabular}

CBCL items to which the content showed a good match to the description provided by the authors of the types (Frick et al. [15]) that were clustered to form four types of externalizing behavior

approach [21] was used to determine developmental trajectories of the four classified externalizing behavior types. In the first five waves of this study, 6,932 observations were collected on which basis the trajectories were computed. For every child, his/her trajectory was determined within each externalizing behavior type.

The semi-parametric mixture model allows for differences between groups in the shape of the developmental trajectories and is also appropriate for analyzing withinsubject-level development. Within the behavior types, the best possible number of groups with different developmental trajectories were estimated and selected using the Bayesian information criterion [21]. We used a ZeroInflated Poisson (ZIP) distribution for estimating the trajectories. Estimation using a ZIP distribution addresses both non-normality and the abundance of zeros typically 

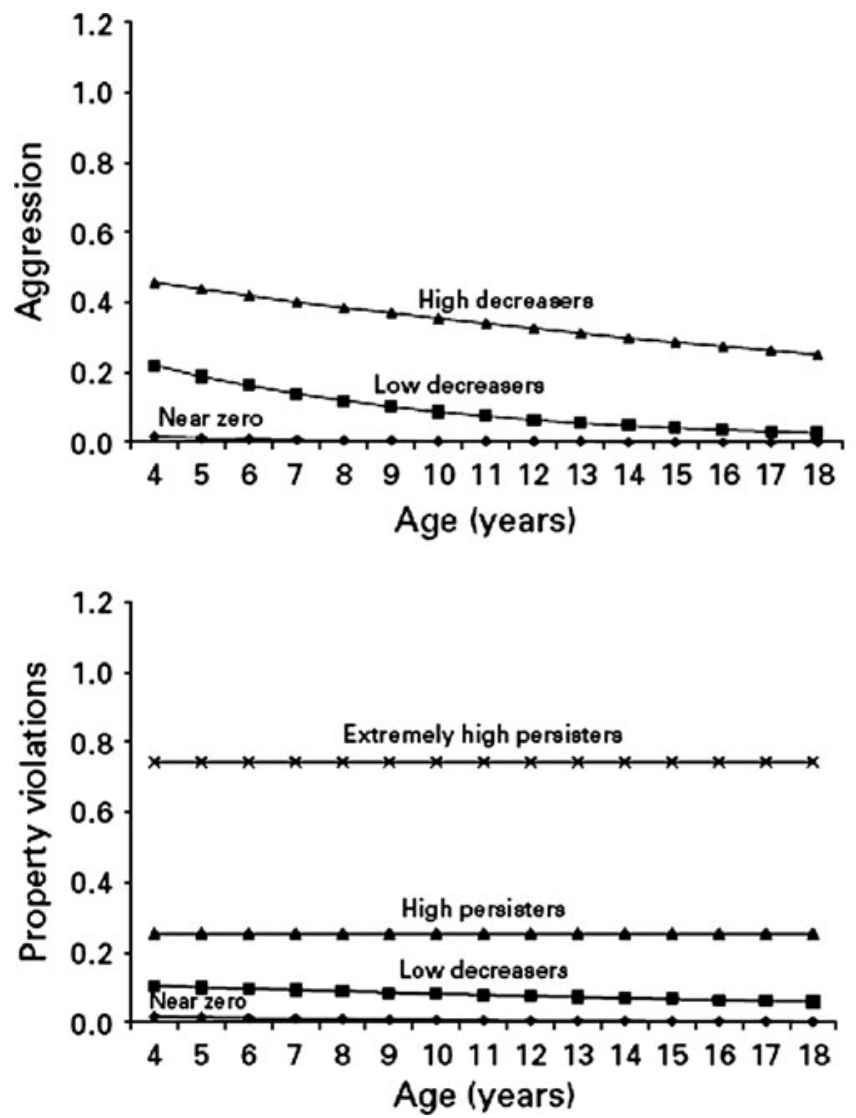

Fig. 2 Developmental trajectories in childhood externalizing behavior types. Group-based developmental trajectories of aggression, opposition, property violations, and status violations. The $y$ axis

found in distributions of externalizing behavior [6, 21]. Model estimation produces two key outputs: parameter estimates that distinguish the shapes of the developmental trajectories [6] and also, for each of the trajectories, the probability of group membership for each individual in the sample. The largest probability for each individual indicated the trajectory that best matched to that individual's behavior over time. With these probabilities, each child was assigned to the trajectory of each externalizing type that best described their individual developmental trajectory. Therefore, each child could be classified at the same time in, for example, a high level trajectory for opposition and a low-level trajectory for aggression. There were equal amounts of younger and older children classified in each trajectory, since there were no age effects in the assignment of the individuals to the trajectories. The child's classifications were used in further analyses.

Three trajectories were found for the externalizing behavior type aggression: a 'near zero' trajectory, a 'low decreasers' trajectory, and a 'high decreasers' trajectory. Six trajectories were found for the behavior type opposition: a 'near zero' trajectory, a 'low decreasers' trajectory,
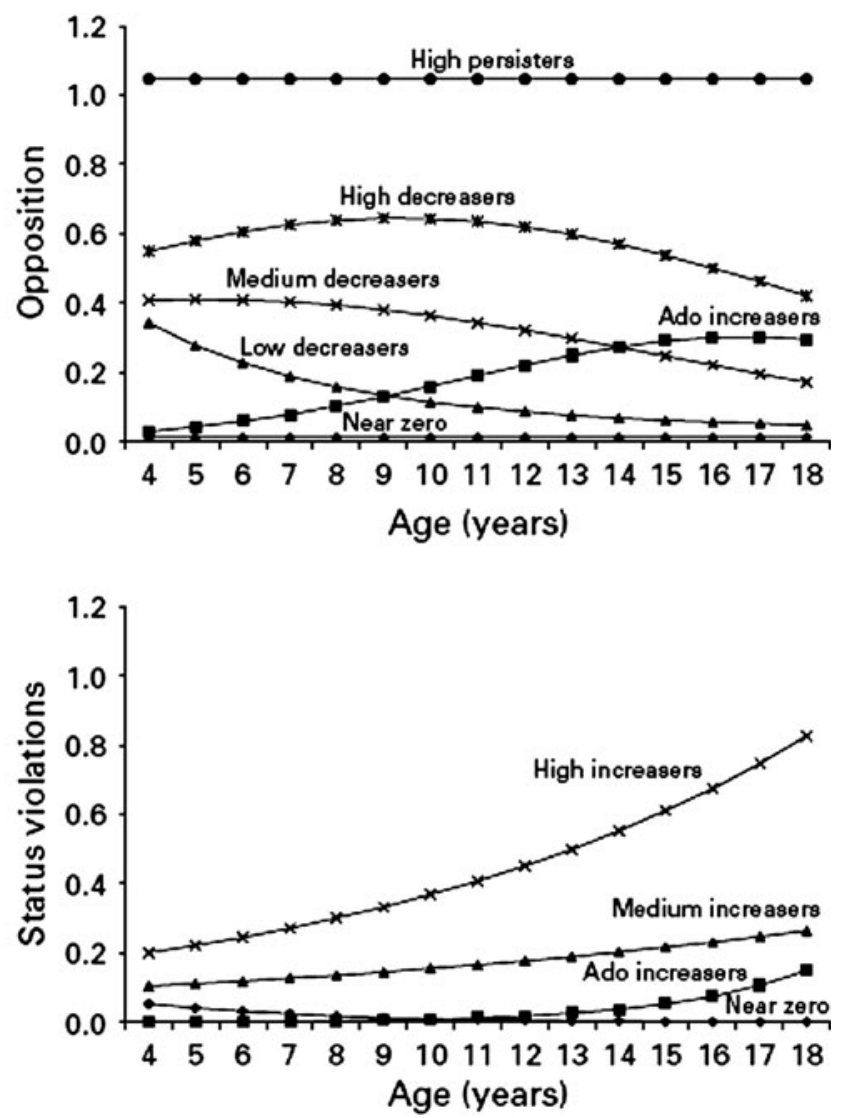

represents the raw syndrome scores. (From Bongers et al. [8]; reprinted with permission of Blackwell Publishing.) "Ado" Adolescence

a 'medium decreasers' trajectory, an 'adolescent increasers' trajectory, a 'high persisters' trajectory and a 'high decreasers' trajectory. Four trajectories were found for property violations: a 'near zero' trajectory, a 'low decreasers' trajectory, a 'high persisters' trajectory, and an 'extremely high persisters' trajectory. In status violations, a 'near zero' trajectory, an 'adolescent decreasers' trajectory, a 'medium increasers' trajectory, and a 'high increasers' trajectory was found.

In the current study, 1,365 adults participated. Of this group of participants, 407 were assigned to a high-level trajectory in at least one type. Of these high-level participants, 302 participated in only one externalizing behavior type in a high-level group, 82 participants in two types, 17 participated in three externalizing behavior types in a highlevel group, and 6 in all four externalizing behavior types. Because the 'extremely high persisters' group of opposition consisted of only two participants, this group was combined with the 'high persisters' group. The number of individuals within each trajectory can be found in Table 2 . No significant differences were found between participants with missing assessments and participants with 
Table 2 Number of participants in the developmental trajectories

\begin{tabular}{lrrl}
\hline $\begin{array}{l}\text { Developmental } \\
\text { trajectory }\end{array}$ & $n$ & $\begin{array}{l}\text { Percentage of } \\
\text { total sample }\end{array}$ & $\begin{array}{l}\text { Percentage } \\
\text { males }\end{array}$ \\
\hline Aggression & 1,473 & 71.0 & 41.7 \\
$\quad$ Near zero & 444 & 21.4 & 65.3 \\
$\quad$ Medium decreasers & 159 & 7.7 & 70.4 \\
$\quad$ High decreasers & & & \\
Opposition & 148 & 7.1 & 43.9 \\
$\quad$ Near zero & 491 & 23.7 & 44.6 \\
Low decreasers & 674 & 32.5 & 50.3 \\
Medium decreasers & 125 & 6.0 & 41.6 \\
$\quad$ Adolescence increasers & 503 & 24.2 & 53.5 \\
High decreasers & 135 & 6.5 & 53.3 \\
High persisters & & & \\
Property violations & 1,548 & 74.6 & 45.4 \\
$\quad$ Near zero & 421 & 20.3 & 56.3 \\
Low decreasers & 107 & 5.2 & 71.0 \\
High persisters & & & 43.7 \\
Status violations & 1,052 & 50.7 & 46.8 \\
Near zero & 485 & 23.4 & 60.5 \\
Adolescence increasers & 514 & 24.8 & 1.2 \\
Medium increasers & 25 & 1.2 & \\
High increasers & & & \\
\hline
\end{tabular}

Number of individuals within each trajectory, percentage of individuals within each trajectory of the total sample, and percentage of males within each trajectory of the total sample

assessments in all five waves on any of the CBCL scales (see [6] for further details of this analysis).

\section{Adult behavioral or emotional problems}

The Adult Self-Report (ASR) [2] is the adult equivalent of the CBCL. It is a questionnaire intended for completion by adults of 18- to 59-year-old and it includes 123 problem items, covering behavioral or emotional problems that have occurred during the past 6 months. The items are scored on a three-point scale: 0 (not true), 1 (somewhat or sometimes true) and 2 (very true or often true). The items can be scored on eight syndrome scales: Withdrawn, Somatic Complaints and Anxious/Depressed (which form the Internalizing scale), Rule-Breaking Behavior, Aggressive Behavior and Intrusive (which form the Externalizing scale), Thought Problems and Attention Problems. A Total Problem Score is calculated by summing the individual item scores. The reliability and validity of the ASR [2] have been confirmed for the Dutch version [34]. In our sample, the ASR had high internal consistency coefficients, i.e. Cronbach's alphas higher than 0.70 (range 0.72-0.89), except for Thought Problems (0.56) and Rule-Breaking Behavior (0.66).
Statistics

\section{Linear regression analyses}

To investigate associations between childhood externalizing developmental trajectories and emotional and behavioral problems in adulthood, we performed multiple linear regression analyses. We tested whether associations existed between all the trajectories in all four externalizing behavior types and adult ASR scale scores. The regression analyses were performed in steps, for every subtype and ASR scale separately. In the first step, we entered the demographic variables gender and SES in the model as predictors, and in the second step, we entered the trajectories of externalizing behavior in the model as predictors. This analysis indicates whether individuals in other trajectories were at higher risk for adverse outcomes than the individuals in the near-zero group. In the third step, we determined for all models, whether there were interaction effects between gender and the separate trajectories. We also tested whether associations between trajectories within every externalizing behavior type and outcomes were significantly different from each other. We reported significant $\beta$ values of the multiple linear regression analyses with the 'near zero' trajectories of each type as reference category, adjusted for gender and SES.

\section{Results}

In the multiple regression analyses, many associations were found between childhood externalizing developmental trajectories and adult problem behavior (Table 3). In general, we primarily found associations between high-level trajectories in the four externalizing behavior types and externalizing behavior in adulthood, and not between lowlevel trajectories and later problem behavior. Developmental trajectories of opposition and status violations showed more associations than the trajectories of aggression and property violations. The high-level and mediumlevel trajectories predicted problems in all the eight syndrome scales in adulthood. Furthermore, the adolescent increasers trajectories of opposition and status violations predicted very few problems in adulthood, and only showed associations with the adult problems in externalizing scales. Overall, the externalizing behavior trajectories predicted mostly aggressive behavior and rule-breaking behavior at follow-up.

Comparison of the regression coefficients of the different trajectories predicting adult outcome within each externalizing behavior type indicated that, first, for all the significant associations of aggression, high decreasers showed significantly stronger associations with adult 


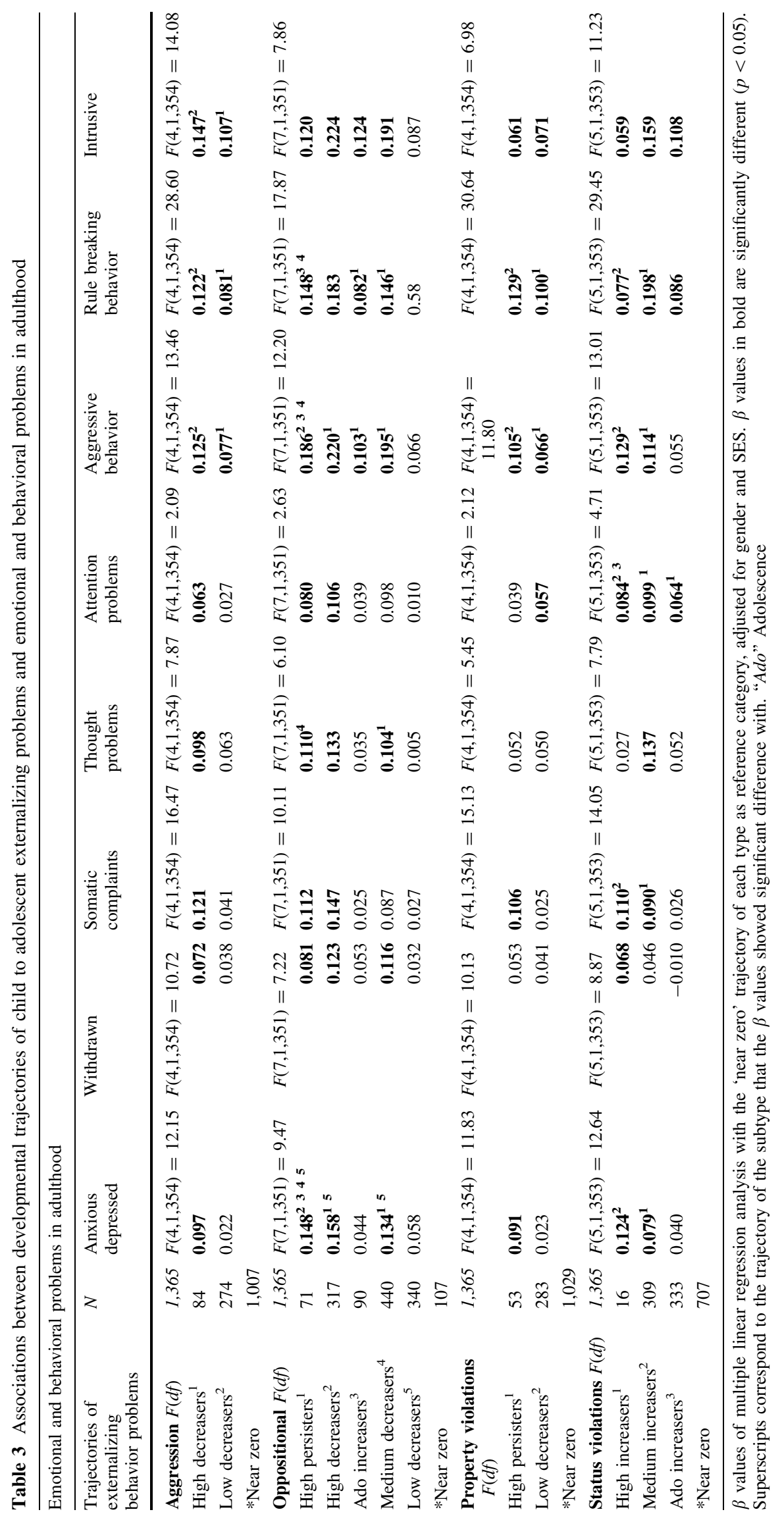


outcome than low decreasers. Second, for opposition, associations between high persisters and high decreasers were significantly different for anxious/depressed and aggressive behavior, and unexpectedly, high decreasers showed a stronger association with adult outcome than the high persisters. In addition, high-level trajectories showed significantly stronger associations with adult outcome than medium-level trajectories in this subtype. Third, as regards the developmental trajectories of property violations, the high persisters showed significantly stronger associations with adult outcome than low decreasers. Finally, for status violations, associations were significantly stronger between high increasers and anxious/depressed, somatic complaints, withdrawn and aggressive behavior. Associations with somatic complaints, thought problems, attention problems, rule-breaking behavior and intrusive problems were significantly stronger for medium increasers.

\section{Discussion}

The aim of this study was to predict adult problems from several child developmental trajectories of four types of externalizing behavior over a period of 24 years in a longitudinal population-based study of 2,076 children. Consistent with previous findings [13, 16, 17, 25, 27], we found that children displaying high levels of externalizing behavior at any time point, and regardless of the course of their problems (i.e. increasing/decreasing/persisting), run a larger risk to have poor outcomes in adult life, compared with children with low levels of externalizing behavior. Furthermore, children displaying externalizing behaviors of the oppositional and status violations types showed a larger variety of psychopathology in adulthood than children displaying externalizing behaviors of the aggressive and property violations types. This prospective community study is unique in terms of its long follow-up time, and its ability to determine the predictive strength of various developmental trajectories in empirically derived types of externalizing behavior on psychopathology in middle adulthood.

In general, and in line with previous studies that investigated the long-term continuity of externalizing behavior $[10,20]$, we found that externalizing behavior problems in childhood showed persistence into adulthood. Trajectories in all externalizing behavior types predicted aggressive behavior, rule-breaking behavior and intrusive problems, which are the three externalizing subscales of the ASR. Even the low-level trajectories of aggression and property violations predicted externalizing behavior in adulthood. Our findings confirm that childhood externalizing behavior shows long-term continuity, even up to middle adulthood.
Apart from predicting externalizing behavior problems in adulthood, severe developmental trajectories of externalizing behavior also predicted adult internalizing problems. Although relatively few population-based studies have reported outcomes in adulthood, heterotypic continuity of childhood externalizing behavior into adult internalizing problems has been previously reported [14]. However, the majority of previous studies indicate that this heterotypic continuity results primarily from the comorbidity between externalizing and internalizing problems in childhood $[5,20,28]$. Hence, a possible reason why externalizing behavior trajectories predict internalizing problems in adulthood in this study could be because we did not control for co-occurring internalizing problems in childhood or adolescence.

Of the four types of externalizing behavior we investigated, oppositional and status violations were most predictive of later emotional and behavioral problems. These results are in line with findings on previous waves of this study [8], and with studies reporting associations between oppositional and adult psychopathology even after control for co-occurring conduct problems $[23,30]$. One possible explanation to these findings could be that these two externalizing behavior types comprise behaviors that are primarily reactive, non-destructive and affective behaviors and entail negative emotionality (e.g. anger, runaway, rule breaking), in contrast to aggression and property violations that primarily comprise proactive and violent behaviors that are offensive and instrumental (e.g. bullying, vandalism). Proactive and reactive aggression have been found to be two distinct subtypes of externalizing behavior with different adult outcomes. Proactive and violent individuals tend to bully and be very unemotional, whereas reactive individuals show impulsive, angry responses to aversive events, particularly perceived by interpersonal threat $[11,36]$. We found that children who show these reactive externalizing problems caused by emotional provocation suffer from later internalizing and attention problems $[12,36]$.

Because previous studies showed that development of externalizing behavior can follow various trajectories [9, $10,18,22,25]$, we used latent class growth analysis to analyze trajectories of externalizing behavior, because this method is well adapted for modeling growth of phenomena within a population in which population members are not following a common developmental process of growth or decline. Given that we analyzed the distinctive trajectories within the externalizing behavior types, we were able to look at the differences in the associations between trajectories and outcomes in adulthood. In accordance with findings of previous studies [20, 24, 25, 38], we found that high-level externalizing trajectories are most predictive for adult problems. In a review of conduct disorder and its outcomes in general population studies it was found that 
increasing severity of externalizing behavior was associated with an increasing risk of an emotional disorder in adulthood [37]. Also, Moffitt et al. [20] investigated the continuity of externalizing behavior and found a severe 'life-course-persistent' trajectory in a sample of externalizing individuals in a follow-up at age 26 years. In a 32 year follow-up of the above sample it was found that individuals that developed through the most severe developmental subtype of DSM-IV conduct problems suffered from the most mental health problems [25]. Taken together, we can confirm that high-levels of externalizing behavior in childhood and adolescence are linked to poor outcomes in adulthood.

Investigating the predictive value of distinct trajectories to problems in adulthood, we found that primarily the level of the trajectories, and not the direction had impact. These findings are consistent with the findings of previous studies in which children in high-level trajectories had more problems in adulthood, independent from the direction of the development $[8,25]$. The present study extends these findings because we were able to report that all high-level trajectories in every type of externalizing behavior showed associations with the majority of the behavioral problems up to the age of 40 years. In addition, the medium-level trajectories, which are still relatively high in severity, showed multiple associations. In general, we can conclude that children who develop along a relatively high-level trajectory are more at risk for adult problems, despite the course of their high-level trajectory.

Longitudinal studies have repeatedly reported a developmental subtype of externalizing behavior in which individuals only suffer from problem behavior during adolescence [9, 20, 24]. In the current study, we did not find significant associations between the adolescent increasers trajectory of status violations and adult problems. This confirms the existence of an adolescence-limited subgroup. However, we also found an adolescent increasers subgroup that did show associations with externalizing behavior; the adolescent increasing trajectory of oppositional predicted all externalizing behavior scales (i.e. aggressive behavior, rule-breaking behavior and intrusive). This contradicts the existence of an adolescent-limited subtype of externalizing behavior. Interestingly, this was also found in a recent study of Odgers et al. [24]. Their results also showed mixed evidence for the existence of the adolescent onset group. Although preliminary, the above findings could indicate that the adolescent-limited subtype does not exist in all types of externalizing behavior, which confirms the importance of examining subtypes of externalizing behavior.

Our findings should be interpreted in light of two limitations. First, although we achieved a relatively high response rate in a 24-year follow-up, a considerable proportion of the original sample from 1983 did not participate in this follow-up. By interpreting our results, one should be aware of the fact that in longitudinal populationbased studies, high-risk people are the most difficult to keep included. Although selective attrition effects were small in the present study, some children with the most severe externalizing behavior problems were not be included. Therefore, results may not generalize to high-risk populations. Consequently, studies on high-risk children are essential to complete the present findings on the predictive value of developmental trajectories of externalizing behavior. Second, the results of this study may have been influenced by time dependent environmental covariates, such as economic growth, ethnic distributions, or family structures that we did not control for.

\section{Conclusion}

Despite the above limitations, this longitudinal study shows a relation between child and adolescent externalizing behavior and adult psychopathology, even over a 24-years time-interval. We can conclude that, high levels of externalizing behavior in childhood and adolescence are associated with poor adult outcome, regardless of the developmental course of these behaviors. Therefore, intervention and prevention should focus on individuals that show severe externalizing problems at any point in childhood or adolescence. Because children and adolescents with externalizing behavior in the oppositional behavior and status violations subtypes were most likely to suffer from adult psychopathology, we recommend that prevention and intervention should focus on children and adolescents showing 'reactive' externalizing behavior (such as anger, temper and runaway) and substance abuse.

\section{References}

1. Achenbach TM (1991) Manual for the child behavior checklist/4 18 and 1991 child profile. Department of Psychiatry, University of Vermont, Burlington

2. Achenbach TM (1997) Manual for the young adult self-report and young adult behavior checklist. Department of Psychiatry, University of Vermont, Burlington

3. Achenbach TM, Dumenci L, Rescorla LA (2001) Ratings of relations between DSM-IV diagnostic categories and items of the CBCL/6-18, TRF, and YSR. University of Vermont, Research center for children, youth, and families, Burlington, Vermont

4. American Psychiatric Association (1994) Diagnostic and statistical manual of mental disorders (4th edn). Washington, DC, USA

5. Angold A, Costello EJ, Erkanli A (1999) Comorbidity. J Child Psychol Psychiatry 40:57-87

6. Bongers IL, Koot HM, van der Ende J, Verhulst FC (2004) Developmental trajectories of externalizing behaviors in childhood and adolescence. Child Dev 75:1523-1537 
7. Bongers IL, Koot HM, van der Ende J, Verhulst FC (2003) The normative development of child and adolescent problem behavior. J Abnorm Psychol 112:179-192

8. Bongers IL, Koot HM, van der Ende J, Verhulst FC (2008) Predicting young adult social functioning from developmental trajectories of externalizing behaviour. Psychol Med 38:989-999

9. Brame B, Nagin DS, Tremblay RE (2001) Developmental trajectories of physical aggression from school entry to late adolescence. J Child Psychol Psychiatry 42:503-512

10. Broidy LM, Nagin DS, Tremblay RE, Bates JE, Brame B, Dodge KA, Fergusson D, Horwood JL, Loeber R, Laird R, Lynam DR, Moffitt TE, Pettit GS, Vitaro F (2003) Developmental trajectories of childhood disruptive behaviors and adolescent delinquency: a six-site, cross-national study. Develop Psychol 39:222-245

11. Dodge KA, Coie JD (1987) Social-information-processing factors in reactive and proactive aggression in children's peer groups. J Pers Soc Psychol 53:1146-1158

12. Dodge KA, Lochman JE, Harnish JD, Bates JE, Pettit GS (1997) Reactive and proactive aggression in school children and psychiatrically impaired chronically assaultive youth. J Abnorm Psychol 106:37-51

13. Fergusson DM, Horwood LJ, Ridder EM (2007) Conduct and attentional problems in childhood and adolescence and later substance use, abuse and dependence: results of a 25-year longitudinal study. Drug Alcohol Depend 88(Suppl 1):S14-S26

14. Fergusson DM, Horwood LJ, Ridder EM (2005) Show me the child at seven: the consequences of conduct problems in childhood for psychosocial functioning in adulthood. J Child Psychol Psychiatry 46:837-849

15. Frick PJ, Lahey BB, Loeber R, Tannenbaum L, Van Horn Y, Christ MAG, Hart EL, Hanson K (1993) Oppositional defiant disorder and conduct disorder: a meta-analytic review of factor analyses and cross-validation in a clinic sample. Clin Psychol Rev 13:319-340

16. Kim-Cohen J, Caspi A, Moffit TE, Harrington H, Milne BJ, Poulton R (2003) Prior Juvenile diagnoses in adults with mental disorder. Arch Gen Psychiatry 60:709-717

17. Mannuzza S, Klein RG, Moulton JL 3rd (2008) Lifetime criminality among boys with attention deficit hyperactivity disorder: a prospective follow-up study into adulthood using official arrest records. Psychiatry Res 160:237-246

18. Moffitt TE (1993) Adolescence-limited and life-course-persistent antisocial behavior: a developmental taxonomy. Psychol Rev 100:674-701

19. Moffitt TE, Caspi A (2001) Childhood predictors differentiate lifecourse persistent and adolescence-limited antisocial pathways among males and females. Develop Psychopathol 13:355-375

20. Moffitt TE, Caspi A, Harrington H, Milne BJ (2002) Males on the life-course-persistent and adolescence-limited antisocial pathways: follow-up at age 26 years. Develop Psychopathol 14:179207

21. Nagin DS (1999) Analyzing developmental trajectories: a semiparametric, group-based approach. Psychol Methods 4:139-157

22. Nagin DS, Tremblay RE (1999) Trajectories of boys' physical aggression, opposition, and hyperactivity on the path to physically violent and nonviolent juvenile delinquency. Child Dev 70:1181-1196

23. Nock MK, Kazdin AE, Hiripi E, Kessler RC (2007) Lifetime prevalence, correlates, and persistence of oppositional defiant disorder: results from the National Comorbidity Survey Replication. J Child Psychol Psychiatry 48:703-713

24. Odgers CL, Caspi A, Broadbent JM, Dickson N, Hancox RJ, Harrington H, Poulton R, Sears MR, Thomson WM, Moffitt TE (2007) Prediction of differential adult health burden by conduct problem subtypes in males. Arch Gen Psychiatry 64:476-484

25. Odgers CL, Moffitt TE, Broadbent JM, Dickson N, Hancox RJ, Harrington H, Poulton R, Sears MR, Thomson WM, Caspi A (2008) Female and male antisocial trajectories: from childhood origins to adult outcomes. Develop Psychopathol 20:673-716

26. Roisman GI, Aguilar B, Egeland B (2004) Antisocial behavior in the transition to adulthood: the independent and interactive roles of developmental history and emerging developmental tasks. Develop Psychopathol 16:857-871

27. Simonoff E, Elander J, Holmshaw J, Pickles A, Murray R, Rutter M, Silberg J, O'Connor T, Meyer J, Maes H (2004) Predictors of antisocial personality: continuities from childhood to adult life. Br J Psychiatry 184:118-127

28. Sourander A, Jensen P, Davies M, Niemela S, Elonheimo H, Ristkari T, Helenius H, Sillanmaki L, Piha J, Kumpulainen K, Tamminen T, Moilanen I, Almqvist F (2007) Who is at greatest risk of adverse long-term outcomes? The Finnish from a Boy to a Man study. J Am Acad Child Adolesc Psychiatry 46:1148-1161

29. Stanger C, Achenbach TM, Verhulst FC (1997) Accelerated longitudinal comparisons of aggressive versus delinquent syndromes. Develop Psychopathol 9:43-58

30. Timmermans M, van Lier PA, Koot HM (2008) Which forms of child/adolescent externalizing behaviors account for late adolescent risky sexual behavior and substance use? J Child Psychol Psychiatry 49:386-394

31. Tremblay RE (2000) The development of aggressive behaviour during childhood: what have we learned in the past century? Int J Behav Develop 24:129-141

32. Van Meurs I, Reef J, Verhulst F, van der Ende J (2008) Intergenerational transmission of child problem behaviors: a longitudinal, population-based study. J Am Acad Child Adolesc Psychiatry 48(2): 138-145

33. van Westerlaak JH, Kropman JA, Collaris JWM (1975) Beroepenklapper (Manual for occupational level). Instituut voor Sociologie, Nijmegen

34. Vanheusden K, van der Ende J, Mulder CL, van Lenthe FJ, Verhulst FC, Mackenbach JP (2009) Beliefs about mental health problems and help-seeking behavior in Dutch young adults. Soc Psychiatry Psychiatr Epidemiol 44:239-246

35. Verhulst FC, van der Ende J, Koot HM (1996) Handleiding voor de CBCL/4-18 (Manual for the CBCL/4-18). Erasmus University/ Department of Child and Adolescent Psychiatry, Sophia Children's Hospital, Rotterdam

36. Vitaro F, Brendgen M, Tremblay R (2002) Reactively and proactively aggressive children: antecedent and subsequent characteristics. J Child Psychol Psychiatry 43:495-505

37. Zoccolillo M (1992) Cooccurrence of conduct disorder and its adult outcomes with depressive and anxiety disorders-a review. J Am Acad Child Adolesc Psychiatry 31:547-556

38. Zoccolillo M, Pickles A, Quinton D, Rutter M (1992) The outcome of childhood conduct disorder: implications for defining adult personality disorder and conduct disorder. Psychol Med 22:971-986 